\title{
Spontaneous Resolution of a Giant Pulmonary Bulla in an Older Woman: Role of Functional Assessment
}

\author{
Simone Scarlata ${ }^{a, b}$ Matteo Cesari ${ }^{a, b} \quad$ Irene Caridi ${ }^{a, b}$ Domenica Chiurco ${ }^{a, b}$ \\ Raffaele Antonelli-Incalzi ${ }^{\mathrm{a}-\mathrm{c}}$ \\ ${ }^{a}$ Centro per la Salute dell'Anziano - Area di Geriatria, Università Campus Bio-Medico, and \\ ${ }^{b}$ Alberto Sordi Foundation, Rome, and ${ }^{~ C}$ Cittadella della Carità Foundation, Taranto, Italy
}

\section{Established Facts}

- Pulmonary bullae usually occur as isolated abnormalities in otherwise normal lung tissue or, more frequently, in the presence of emphysema. They tend to progressively increase in their size, and spontaneous regressions have very rarely been reported.

- The impact on physical performance and functional status following the spontaneous resolution of a giant pulmonary bulla has never been assessed in previous reports.

\section{Novel Insights}

- This case report shows the unusual evolution of a giant pulmonary bulla and (likely more important) how a simple index of physical performance like the 6-min walking test, different from a complete spirometry, could capture its effects on health status.

\section{Key Words}

Pulmonary bulla - Elderly $\cdot$ Functional assessment

\begin{abstract}
Pulmonary bullae usually occur as isolated abnormalities in otherwise normal lung tissue or, more frequently, in the presence of emphysema. Pulmonary bullae tend to progressively increase in their size, and spontaneous regressions have very rarely been reported. In this paper, we report the case of an older woman experiencing the spontaneous resolution of a giant pulmonary bulla. Interestingly, the reported case
\end{abstract}

\section{KARGER}

Fax +41613061234 E-Mail karger@karger.ch www.karger.com
(C) 2010 S. Karger AG, Basel

0025-7931/11/0811-0059\$38.00/0

Accessible online at:

www.karger.com/res is characterized by a negligible improvement in respiratory function parameters, despite a substantial increase in the 6-min walking test and the oxygen arteriolar partial pressure.

Copyright $\odot 2010$ S. Karger AG, Basel

\section{Introduction}

Pulmonary bullae usually occur as isolated abnormalities in otherwise normal lung tissue or, more frequently, in the presence of emphysema. Pulmonary bullae tend to 

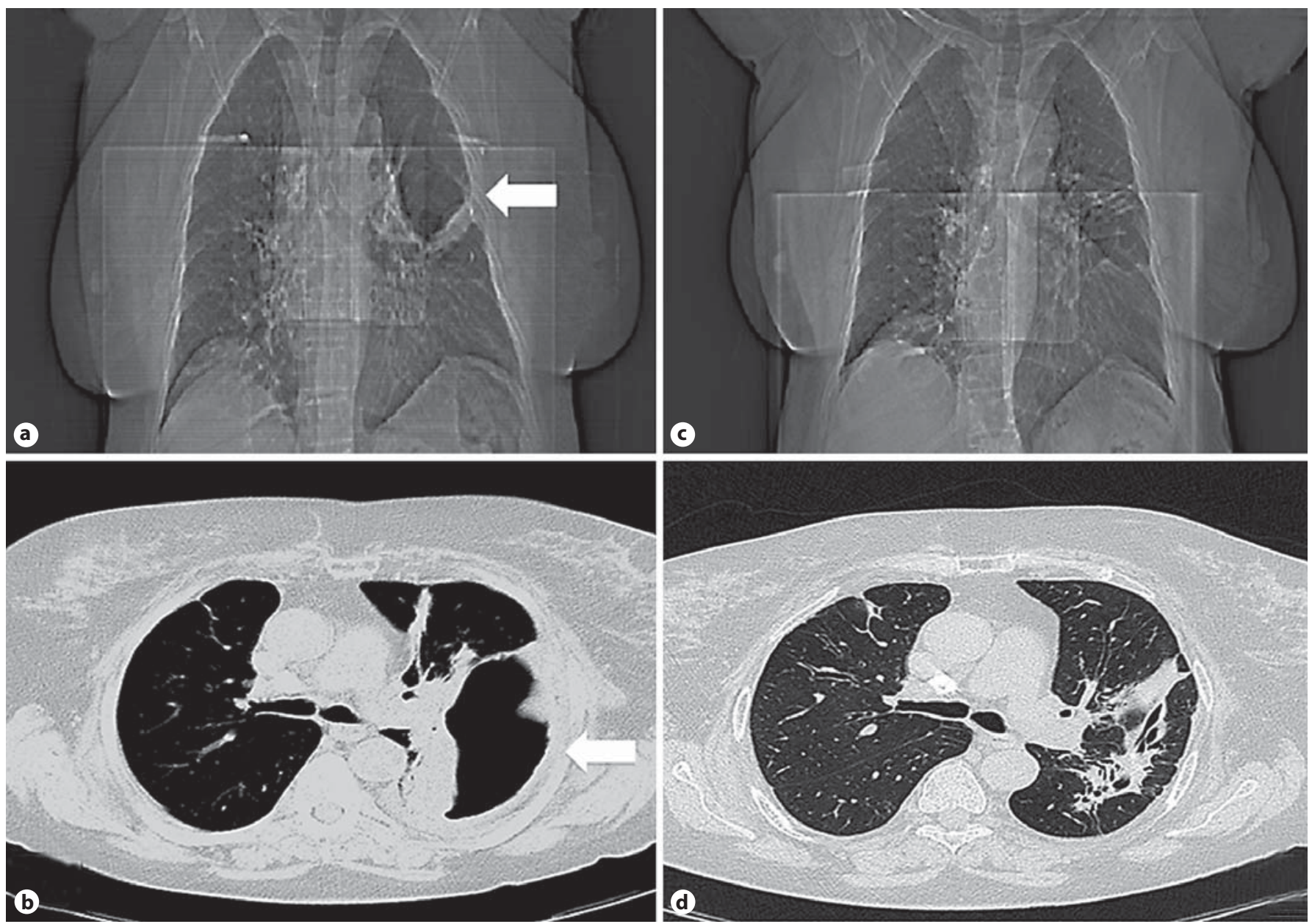

Fig. 1. Chest CT scans performed in December 2006 (a and b) showing a giant bulla in the left upper lobe with surrounding atelectasis and in April 2009 (c and d) showing a substantial reduction in its size.

progressively increase in their size, and spontaneous regressions have very rarely been reported. In this paper, we report the case of an older woman experiencing the spontaneous resolution of a giant pulmonary bulla. Interestingly, the reported case is characterized by a negligible improvement in respiratory function parameters, despite a substantial increase in the 6-min walking test and the oxygen arteriolar partial pressure.

\section{Case Report}

A 75-year-old woman was admitted to the Geriatric Acute Care Unit of the Health Center for Elderly People at the Campus Bio-Medico University Hospital (Rome, Italy) in April 2009 for a re-evaluation of the chronic HCV-related liver disease she had been suffering for several years. The patient, with a 45-pack-year smoking history, reported a 2-year history of giant bullous emphysema. She also had osteoporosis with severe kyphoscoliosis, obesity, urge urinary incontinence and major depressive disorder. She was on antidepressive treatment (with a serotonin-noradrenalin reuptake inhibitor, Venlafaxine), and aerosol therapy with long-acting $\beta_{2}$-agonist, anticholinergic and corticosteroid.

About 2 and a half years earlier (December 2006), the patient had undergone a chest computed tomography (CT) scan documenting an $11 \times 9 \mathrm{~cm}$ wide giant bulla in the upper left lobe, clearly compressing the surrounding parenchyma with consequent regional atelectasis and partial mediastinic dislocation (fig. la and b). A spirometry test, performed at that time, documented a severe restrictive ventilatory pattern with no sign of bronchial obstruction and air trapping (table 1). Carbon monoxide lung diffusion after correction for alveolar volume was moderately decreased. At that time, the patient did not report sudden onset of fatigue or severe dyspnoea (even under stress). Asthenia and muscle fatigue, but not dispnoea, were responsible for a limited physical performance of $189 \mathrm{~m}$ ( $45 \%$ of predicted) in the 6 -minute walking test. Chronic 
Table 1. Pulmonary function tests at spirometry and lung volumes obtained by He dilution technique before and after spontaneous resolution of the pulmonary bulla

\begin{tabular}{|c|c|c|}
\hline & December 2006 & April 2009 \\
\hline FEV1, 1 & $0.98(54)$ & $1.08(61)$ \\
\hline FVC, 1 & $1.19(53)$ & $1.32(61)$ \\
\hline FEV1/FVC & 82.8 & 81.5 \\
\hline $\mathrm{RV}(\mathrm{He}), 1$ & $1.03(50)$ & $1.18(56)$ \\
\hline $\mathrm{FRC}(\mathrm{He}), 1$ & $1.12(42)$ & $1.40(53)$ \\
\hline TLC (He), 1 & $2.22(47)$ & $2.50(53)$ \\
\hline $\mathrm{RV} / \mathrm{TLC}$ & 106 & 106 \\
\hline $\mathrm{Tl}(\mathrm{CO}), \mathrm{ml} /(\mathrm{min} \times \mathrm{mm} \mathrm{Hg})$ & $1.83(9)$ & $3.22(19)$ \\
\hline $\mathrm{K}(\mathrm{CO}), \mathrm{ml} /(\mathrm{min} \times \mathrm{mm} \mathrm{Hg})$ & $2.33(41)$ & $3.85(69)$ \\
\hline $\mathrm{PaO}_{2}, \mathrm{~mm} \mathrm{Hg}$ & 60.8 & 72 \\
\hline $\mathrm{PaCO}_{2}, \mathrm{~mm} \mathrm{Hg}$ & 35.7 & 47 \\
\hline $\mathrm{PaO}_{2}(\mathrm{~A}-\mathrm{a}), \mathrm{mm} \mathrm{Hg}$ & 47.8 & 39.1 \\
\hline $6 \mathrm{MWD}, \mathrm{m}$ & $189(45)$ & $248(59)$ \\
\hline Borg dyspnoea scale score & 5 & 5 \\
\hline
\end{tabular}

Figures in parentheses are percent of predicted. FEV1 = Forced expiratory volume in $1 \mathrm{~s} ; \mathrm{FVC}=$ forced vital capacity; RV = residual volume; $F R C=$ functional residual capacity; TLC $=$ total lung capacity; $\mathrm{Tl}(\mathrm{CO})=$ carbon monoxide transfer factor; $\mathrm{K}(\mathrm{CO})$ = carbon monoxide transient coefficient; $\mathrm{PaO}_{2}=$ oxygen arteriolar partial pressure; $\mathrm{PaCO}_{2}=$ carbon dioxide arteriolar partial pressure; $\mathrm{PaO}_{2}(\mathrm{~A}-\mathrm{a})$ = oxygen arteriolar-alveolar gradient; $6 \mathrm{MWD}=6$-min walking test distance.

cough, sputum production and/or wheezing were not present. Based on the results of the pulmonary function tests the aerosol therapy was suspended. The spirometry test repeated after pharmacological wash-out confirmed previous findings. Although the presence of a giant bulla may have surgical indication, the patient refused to undergo surgery because of the severely depressed mood.

During the following years, the patient had been attending our outpatient Respiratory Clinic for periodic respiratory function evaluations. At the April 2009 admission to our department, the patient underwent new pulmonary function tests and chest CT scan. At the clinical assessment, she only reported that a mild, transitory and self-resolving left posterior chest pain episode had occurred few weeks earlier. Pulmonary volumes, flows and diffusion capacity showed a mild increase in all lung volumes compared to the results reported in the most recent tests (consistent with those performed in 2006). Surprisingly, the chest CT scan (fig. $1 \mathrm{c}$ and d) documented a dramatic reduction in the bulla dimensions (maximum diameter $1.5 \mathrm{~cm}$ ) and resolution of the previously documented atelectasis. The blood gas analysis showed significant improvements in all the gas exchange parameters, including the oxygen alveolar-arteriolar gradient. The 6 -min walk test showed a $\sim 25 \%$ improvement in physical performance compared to the results obtained in December 2006. The patient was discharged in fairly good general and respiratory conditions, and scheduled for periodic re-evaluations. After 6 months, she was clinically stable and her pulmonary function unmodified. She provided written informed consent to this case report.

Spontaneous Resolution of a Giant Pulmonary Bulla

\section{Discussion}

Pulmonary bullae are pathological air space dilations $>2 \mathrm{~cm}$ in diameter, occurring distally at the terminal bronchioles [1]. They are conventionally defined as 'giant bullae' when they occupy over half of a whole hemithorax volume [2]. The natural history of pulmonary bullae is unpredictable. They commonly tend to gradually increase in size, worsening pulmonary function and often requiring surgical bullectomy. The mechanisms leading to the very rare spontaneous resolution of the pulmonary bullae are unclear and still debated. In our case, the exact (period of) time for the resolution of the bulla cannot be definitely established. It is possible that it suddenly occurred when the patient felt a mild, transitory and selfresolving chest pain, likely due to the rupture of the air space dilation. However, an event like this might be responsible for the onset of pneumothorax (and its consequences), which we did not report. Some of the previously described cases were associated or likely due to lung infections, cough, sputum production and presence of air/fluid level on chest X-ray [3]. It is possible that the resolution of such underlying conditions may facilitate the re-absorption of the bulla. In our case, none of the above-mentioned conditions were present at the first evaluation nor during the follow-up. Nevertheless, we cannot categorically exclude that our CT findings were indeed showing a bullitis (i.e. the superinfection of a bulla). In fact, this condition is usually characterized by the thickening of the bulla walls at the CT scan (a detail which might be, at least partially, found at the first tomography). Among other explanations of the bulla resolution we described, it is worth mentioning that inflammatory mechanisms and clinical conditions responsible for bronchiolar communication obstruction (i.e. neoplasms, mucous plugging, blood clots, anaerobic infections) may lead to the bulla shrinkage through air and fluid absorption [4-6].

Differently from case reports describing similar episodes in patients with obstructive respiratory conditions $[4,5]$, our patient was characterized by an extra-parenchymal restrictive pattern (due to obesity and severe kyphoscoliosis). Nevertheless, the lung re-expansion occurring at the resolution of the bulla was similar to a previously published case [4] and, interestingly, not associated with parallel improvements of the lung volumes (i.e. vital capacity, functional residual capacity and total lung capacity). This phenomenon apparently contradicts the common pathophysiologic explanations, also taking into account the helium dilution technique we adopted (sys- 
tematically underestimating lung volumes due to the inability of gas to diffuse through unventilated areas), instead of the body plethysmography (measuring total lung capacity independently of ventilation). At the same time, significant improvements in all the other measured pulmonary, e.g. gas exchange efficiency and pulmonary blood flow, and physical performance, e.g. 6-min walking test, parameters was present (table 1). It is possible that the conditions accounting for extra-pulmonary restriction prevented lung volume expansion following the resolution of the bulla. On the other hand, the collapse of the bulla likely improved the chest wall mechanics and, then, the ventilation by reducing the muscular workload. Moreover, the improvement of the gas exchange parameters (i.e. the oxygen arteriolar partial pressure, the oxygen arteriolar-alveolar gradient, the carbon monoxide transfer factor and the carbon monoxide transient coefficient) can be explained by a better pulmonary ventilation/perfusion ratio or microvascular recruitment following the lung re-expansion with consequent resolution of possible pre-existing shunts [6].
The lack of improvement in dyspnoea might be explained by the severe major depressive disorder and the patient's sedentary status. However, we believe the most significant finding of our case is related to physical performance, as assessed by the 6-min walking test. To our knowledge, this is the first case report objectively documenting the improvement in physical performance after the spontaneous resolution of a giant pulmonary bulla. Considering the strong relationship existing between physical performance and quality of life, this datum is of particular interest. Consistent with our findings, Bradshaw et al. $[4,5]$, who previously described the only clinical case similar to ours, reported the subjective evaluation that proxies made of the patient as 'having a lot more energy' than usual in those days.

In conclusion, this case report shows the unusual evolution of a giant pulmonary bulla and, likely more important, how a simple index of physical performance like the 6-minute walking test, different from a complete spirometry, could capture its effects on health status.

References

Vita ML, Van Bouwel EL, Bouhouch A, Hendriks JM, Lauwers PR, Van Schil PE: Giant calcified bulla compressing the mediastinum. Respiration 2009;77:331-332.

2 Ciba Guest Symposium: Terminology, definitions, and classification of chronic pulmonary emphysema and related conditions: a report of the conclusions of a Ciba Guest Symposium. Thorax 1959;14:286-299.

3 Goodman RB, Lakshminarayan S: Images in clinical medicine: inflammatory autobullectomy. N Engl J Med 1996;334:1372-1373.
-4 Bradshaw DA, Murray KM, Amundson DE: Spontaneous regression of a giant pulmonary bulla. Thorax 1996;51:549-550.

-5 Stanescu D, Veriter CL: Spontaneous regression of a giant pulmonary bulla. Thorax 1996;51:1283.

- 6 Soni R, McKeough ZJ, Dobbin CJ, Youngh IH: Gas exchange and exercise tolerance following bullectomy. Respirology 2005; 10 : $120-123$. 